\title{
A Modelica Library for Modelling of Electrified Powertrain Digital Twins
}

\author{
Nikolaos Fotias ${ }^{1}$ Ran Bao ${ }^{2}$ Hui Niu ${ }^{2}$ Michael Tiller ${ }^{3}$ \\ Paul McGahan ${ }^{1} \quad$ Adam Ingleby $^{2}$ \\ ${ }^{1}$ Ricardo Prague s.r.o., Czech Republic, \{Nikolaos.Fotias, \\ Paul.McGahan \} ericardo.com \\ ${ }^{2}$ Ricardo UK Ltd., UK, \{Ran.Bao, Hui.Niu, \\ Adam. Ingleby \} ericardo.com \\ ${ }^{3}$ Ricardo Inc., USA, Michael. Tiller@ricardo.com
}

\begin{abstract}
In this paper, a Modelica library of electrified powertrain components is presented and its applications discussed. This library is used to construct digital twins of electrified powertrains during product development. These digital twins provide value by reducing development time and cost, while once the product is in-service, they enable improved condition monitoring. The library includes a multi-fidelity and multi-scale battery and power electronics sub-library, an Electrical Drive Unit (EDU) sub-library modelling different types of electrical machines, and an electrified propulsion system sub-library of template models that leverage the battery, power electronics and EDU components found in the other sublibraries. Finally, an example of applying the proposed library to electrified vehicle development is presented.
\end{abstract}

Keywords: Modelica, Digital Twins, Electrified Powertrain

\section{Introduction}

Digitalisation is revolutionising the complete product lifecycle: from development and production to testing, inservice maintenance and recycling. "Digital Twin" (DT) technology will bring a significant reduction in electric powertrain development time, cost and risk: through upfront design analysis, optimisation and testing in a virtual environment, without the need for multiple prototypes. At its core, a DT is a representation of a physical product that can be used as a testing ground for monitoring, simulating and optimizing design and operational performance.

This paper describes the work done in a project which had the key objective of assessing the impact of DT techniques on product development. In this project, the first focus was developing Digital Twins for each sub-system (Battery, Power Electronics and EDU) in the electrified powertrain. The second focus was the integration of each sub-system, to create an Electrified Powertrain Digital Twin. Such a system level Digital Twin can be used, as part of the virtual product development process, in the design and optimisation of the electrified powertrain.

We have created an Electrified Powertrain Modelica library, called ePropulsionSystem, which incorporates necessary plant models to enable development of Digital Twins. The novelty of this developed library arises from the ability to seamlessly swap between model variants and fidelity levels, while offering an interface to couple with already existing libraries to streamline concept development, with the ultimate goal of effectively linking the developed virtual model to the physical model.

In this paper we will discuss the various models included in the different sub-libraries as well as an example of applying the ePropulsionsystem model to an Electric Vehicle (EV) use case.

\subsection{Literature Review}

The battery models describe not only the electrical behaviour of the battery but also the thermal response and aging characteristics (Einhorn, et al., 2011; Gerl, Janczyk, Krüger, \& Modrow, 2014; Surewaard, Karden, \& Tiller, 2003; Dao \& Schmitke, 2015; Bao, Fotias, \& McGahan, 2021). The most common representations of battery electrical behaviour are electrochemical models and Equivalent Circuit Models (ECM) (Fan, Pan, Bartlett, Canova, \& Rizzoni, 2014; Perez, Shahmohammadhamedani, \& Moura, 2015; Guo, Jin, \& White, 2017; He, Xiong, \& Fan, 2011; Chen \& RinconMora, 2006). It is well known that the overall battery pack performance is influenced significantly by the battery temperature (McGahan, Rouaud, \& Booker, 2019) which we model using a network of 1D lumped thermal components (Pesaran, 2002; Johnson, Pesaran, \& Sack, 2000; Park \& Jaura, 2003; Nelson, Dees, Amine, \& Henriksen, 2002). Investigation of battery aging mechanisms is currently a hot topic in both academia and industry. Calendric ageing and cyclic ageing are two commonly used ageing model. The cause and effect of various battery ageing mechanisms is discussed in detail 
in (Vetter, et al., 2005). Estimating the battery parameters from measured data is also an important feature in several battery libraries (Gerl, Janczyk, Krüger, \& Modrow, 2014; Dao \& Schmitke, 2015; Qin, Li, Wang, \& Zhang, 2019).

To enable easy model parametrisation and development, while still ensuring high fidelity, behavioural modelling was used to develop the components of the power electronics library. The work was based on concepts presented in the literature (Denz, Schmitt, \& Andres, 2014; Cellier, Clauß, \& Urquía, 2007; Lai, Hill, \& Suchato, 2019; Urkizu, et al., 2019), where the response of the system is an amalgamation of the static response and an estimation of the losses, either using analytical solutions, measurement data or the dynamic model of the system. Given the dependence of the device response on the temperature, the developed models were enhanced to capture that behaviour and thermal models for the devices were developed in accordance with (AG, 2020).

The five most common electric machine technologies among Hybrid Electric Vehicles (HEVs) and EVs are Induction Machines (IMs), Switched Reluctance Motors (SRMs), wound-rotor Synchronous Machines (SMs), Direct Current (DC) machines, and Synchronous Permanent Magnet Machines (SMPM) (Bazzi, 2013; Dorrell, Knight, Popescu, Evans, \& Staton, 2010) each with their own advantages and disadvantages. In late 2020, Tesla pioneered a hybrid motor type in the Tesla Model 3, combining characteristics of Interior Permanent Magnet (IPM) motors and Synchronous Reluctance Motors (SynRM) to form the hybrid IPM-SynRM motor.

While an IPM machine demonstrates high efficiency at high speed, this comes at the cost of output torque (Hwang, Han, Kim, \& Cha, 2018). By comparison, IPMSynRM devices possess better efficiency at low speed and better thermal efficiency than traditional SynRMs subject to temperature limits (Ramakrishnan, Stipetic, Gobbi, \& Mastinu, 2018; Xing, Sun, \& Lei, 2014; Lee, Kim, Jung, Hong, \& Kim, 2012; Haumer \& Kral, Motor Management of Permagnent Magnet Synchronous Machines, 2012). Since the IPM is a type of SMPM, this project focused on the development of SMPM and SynRM (abbreviated as SMR in Modelica nomenclature) motors.

There are several software packages available for the mathematical modelling and simulation of EVs and EDUs, including MATLAB/Simulink, Simpower, Python based models and Modelica (Mohd, Hassan, \& Aziz, 2015; McDonald, 2012). Because the Modelica Standard Library (MSL) is open source and provides a library of multi-domain physical models found in automotive components, the MSL can be an excellent starting point for the development of EDU digital twins (Einhorn, et al., 2111). The MSL has a basic electric machine library which includes the traditional asynchronous Induction
Machines and Synchronous Machines (Ceraolo, 2015). The MSL and other Modelica libraries also have extended models that consider friction losses and include thermal effects, e.g., the Fundamental Wave library (Kral \& Haumer, 2011), Advanced library (Haumer, Kral, Kapeller, Bäuml, \& Gragger, 2009) and SmartDrive library (Gragger, Kral, Hansjörg, \& Pirker, 2006).

\section{Library Structure}

Figure 1 shows the typical architecture of the digital twin models created using our ePropulsionSystem library. As shown in Figure 1, the Battery, Power Electronics and EDU are the key components being modeled. This library does not include models of the controllers for these devices as we have chosen to focus exclusively on plant models in this library. The ePropulsionsystem library is structured as shown in Figure 2.

\subsection{Battery}

The key models in the Battery sub-library are the cell level Electrical and Ageing models. These cell level models are then leveraged in the BatteryPack models. These models are fully developed and will be discussed in detail later in this paper. The Battery sub-library also includes Thermal, Cooling, Ancillaries and BatteryManagementSystem component libraries which will be developed in the future and will not be discussed in this paper.

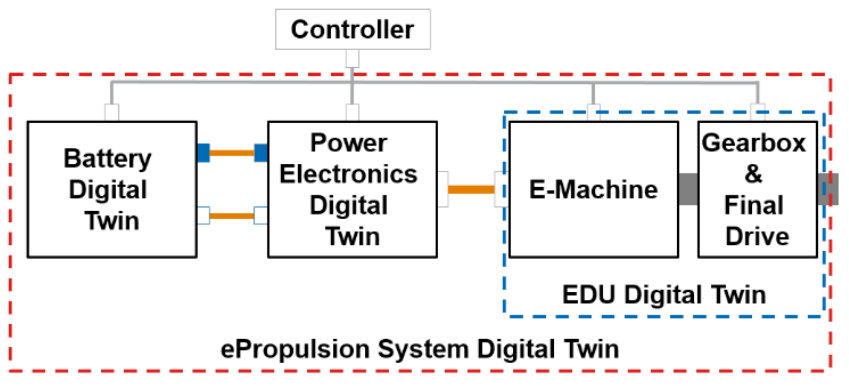

Figure 1. Architecture of a typical ePropulsionsystem digital twin model

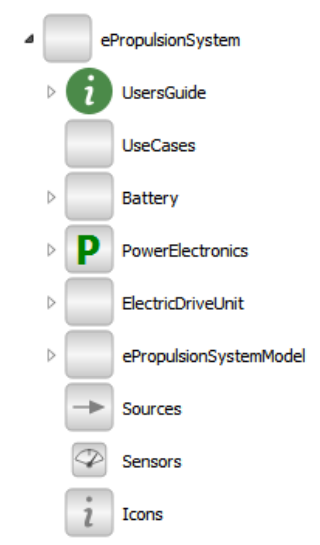

Figure 2. ePropulsionsystem library structure 


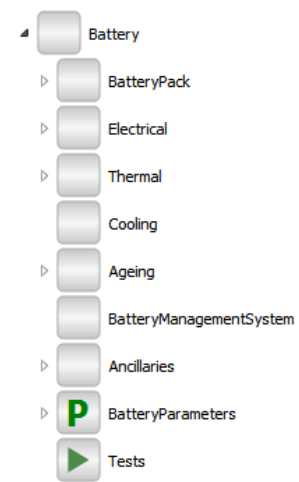

Figure 3. The structure of the Battery sub-library in ePropulsionsystem library

\subsubsection{Electrical}

At present, there are three different electrical cell models included in the Battery sub-library. These are the Ideal Voltage Source Model, Internal Resistance Model, and RC Element Model. Schematics for each of these models are shown in Figure 4. Each of these electrical cell models can be characterized by how their Open Circuit Voltage $(\mathrm{OCV})$ and internal resistance are modeled. For this reason, a template model for electrical cells was created and is shown in Figure 5.
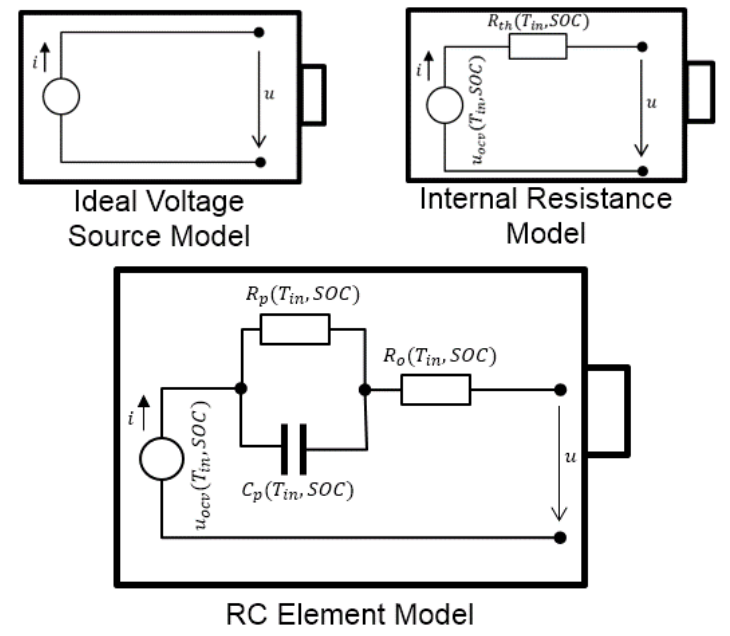

Figure 4. Different electrical cell models included in the Battery sub-library

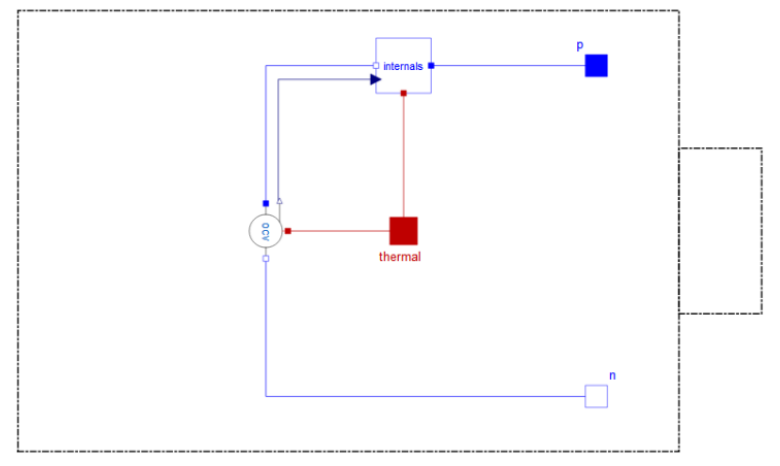

Figure 5. ElectricalCellTemplate model
The Electrical Cell Model Template includes a positive connector ( $\mathrm{p}$ ), a negative connector ( $\mathrm{n}$ ), a thermal connector (thermal) as well as the OCV and internal resistance component models. To use one template to represent three different cell electrical models, the OCV and internal resistance component models are made replaceable. So, a Modelica package, called Electricalinterfaces, was created which includes the interfaces for the OCV and internal resistance components. Different OCV and internal resistance models, which extend from the partial models in the Electricalinterfaces package, were built and included in a package called Electricalcomponents. The structure of an electrical cell model in the Battery package is shown in Figure 6.

In ElectricalComponents, two different OCV models were developed. The first model, Idealocv, uses a constant voltage source for the open circuit voltage. The other model, TzTableOCV, computes the OCV based on the cell temperature and State-of-Charge (SOC). The cell SOC is also calculated in the TzTableOCV model by integrating the current flow in and out of the cell.

Three different models of the internal resistance of the cell were developed. These are the Idealshort model, the TzTableR, and the TzTableRCR model. All of these models are found in the Battery.ElectricalComponents sub-library. The Idealshort model assumes there is no internal resistance in the cell. The TzTableR and TzTableRCR models compute the internal resistance of the cell based on cell temperature and SOC. The topology of the TzTableR features only a single (temperature and SOC dependent) resistance while the topology of the TzTableRCR includes two resistors and a capacitor (all of which depend on temperature and SOC).

As shown in Figure 4, by starting with the ElectricalCell Template model and picking different $\mathrm{OCV}$ and internal resistance sub-models, a number of different electrical cell models can be created.

In this way, using the Electricalcelltemplate model, the three pre-defined electrical cell models, shown in Figure 6, were created: IdealCell, R_Cell and RC_Cell.

\subsubsection{Ageing}

Normally, battery ageing models includes two modes of ageing. These are cycling and calendaring. In this library, only the cycling ageing model is considered. A cycling ageing model is a semi-empirical model of two main effects of ageing on cell performance, capacity fade and power fade. 
It has been shown experimentally that the capacity fade can be described using a power law with energy throughput and is related to temperature via an Arrhenius relationship. This means that the capacity loss (capacity fade), can be described using the equation below (Andrea Cordoba-Arenas, 2015):

$$
Q_{\text {fade }}=k_{Q_{\text {sev }}} A h^{z}
$$

Where, $Q_{\text {fade }}$ is the capacity fade [\%]; $k_{Q_{\text {sev }}}$ is the capacity fade severity factor [-]; Ah is the charge throughput [kAh]; $\mathrm{z}$ is the power exponent [-].

The resulting increase in internal resistance can be described using a very similar equation to capacity fade, however without the power exponent (Andrea CordobaArenas, 2015):

$$
I R_{\text {inc }}=K_{I R_{\text {sev }}} A h
$$

Where, $I R_{\text {inc }}$ is the increase in internal resistance [\%]; $K_{I R_{s e v}}$ is the increase in internal resistance severity factor [-]; Ah is the charge throughput [kAh].

The Ageing model was created by using Equation (1) and (2) and added to the ElectricalCellTemplate model to create a new template called ElectricalCellsWithAgeing as shown in Figure 7. As the Ageing model reduces the cell capacity, and increases the cell internal resistance, the Electricalinterfaces package was updated by adding new inputs and outputs to create a new package called ElectricalinterfacesWithAgeing in the Ageing package; and the ElectricalComponentsWithAgeing was updated to include the calculation of ageing effects from the ElectricalComponents package. The structure of the Ageing package is presented in Figure 6. Again, we can choose models for the OCV and internal losses to create several different electrical cell models some of which are shown in Figure 6.

\subsubsection{BatteryPack}

Using the electrical cell model interface, we can then construct a battery PackModel which is composed of battery cells connected both in series and in parallel. This battery PackModel and its parameters are shown in Figure 8.

In the PackModel, the number of battery cells connected in series and the number of battery cells in parallel are defined by two parameters, ns and np, respectively Using these parameters in conjunction with the array and looping capabilities in Modelica we are then able to automatically generate the serial and parallel connections needed to wire together every cell in the PackModel. Different electrical cell models (with or without ageing) from the Electrical and Ageing package can be chosen as the replaceable CellModeltype model which is used to instantiate each cell in the PackModel. Through our template models we can create both topological and parametric variants to match the underlying cells as shown in Figure 3. We place these fully parameterized models in the Battery.Parameters library. In this way, we can create pack models using any of the parameterized cell models that we have developed (with or without ageing) and independently specify the pack topology as well.

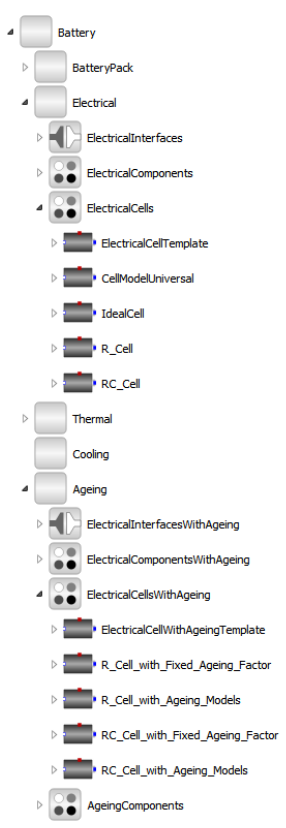

Figure 6. The structure of Electrical and Ageing packages

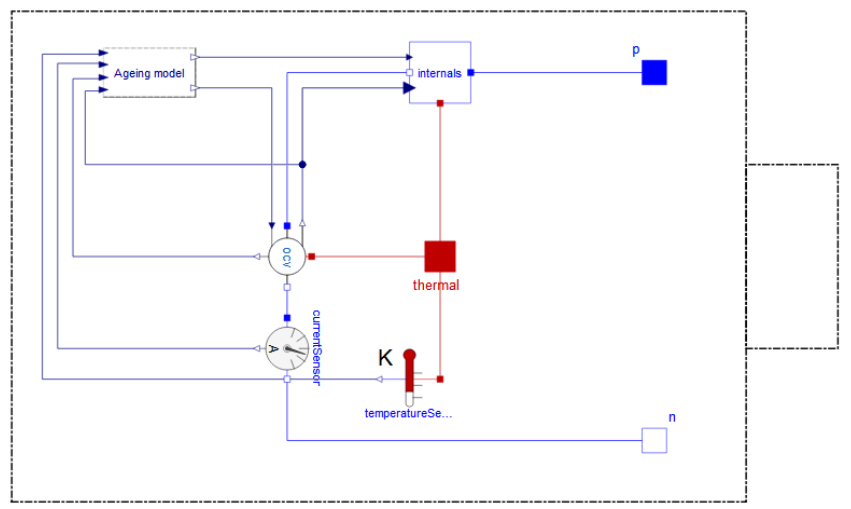

Figure 7. ElectricalCellswithAgeing template 


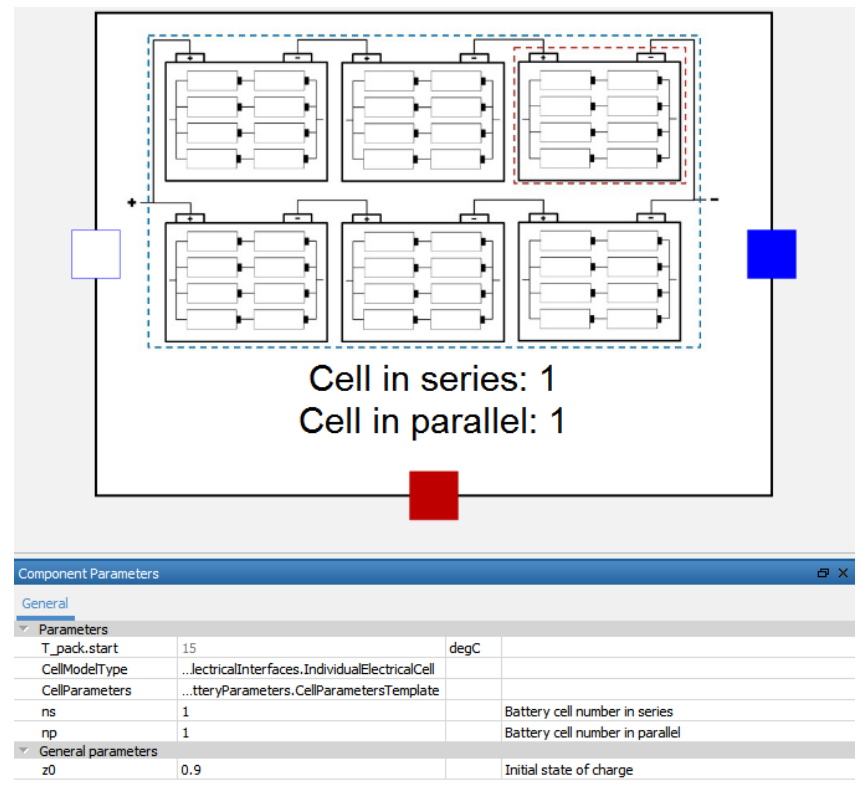

Figure 8. Battery PackModel and its parameters

\subsection{PowerElectronics}

The PowerElectronics sub-library is a part of the overall Digital Twin for Electrified Powertrains library, ePropulsionsystem, and focuses on capturing the behavior of the most commonly used electronic components and topologies, while ensuring easy parametrization from available manufacturer data.

The main focus of the library is the modelling of the behavior of Diodes, Insulated Gate Bipolar Transistors (IGBTs) and Metal-Oxide-Semiconductor Field Effect Transistors (MOSFETs) in a way that enables the user to simulate a variety of electronics topologies utilizing any available parametrization data. For that purpose, the developed models are made up of numerous variants to estimate the static response (static model variants) as well as the corresponding losses of the device in question (loss model variants).

The static model variants for the different semiconductors capture the response of the device disregarding the dynamic behavior due to the presence of capacitances and inductances. The models were developed and organized according to the level of detail that is needed for their parametrization:

- Ideal models, where the devices under investigation are represented by their ideal equivalent circuit.

- Constant models, where parameters such as channel resistance are not affected by system variables.

- Look-up table-based models, where the device parameters at each simulation step are the output of a system variable dependent look-up table.
Such variables include device temperature, gate voltages etc.

All these variants are developed using the same underlying interface and can be seamlessly swapped using replaceable models.

The loss model variants estimate the conduction losses as well as the switching losses of the devices, given a selected static model and the data available to the user. The variants developed are the following:

- Lossless models, which are implemented for fast simulations where heat dissipation is of no interest.

- Constant models, where parameters such as energy release during a switching event are not affected by system variables.

- Look-up table-based models, where parameters, such as the switching energy loss, are estimated at every simulation step from the device variables using look-up tables. Such variables are the device current, the blocking voltage etc.

- Dynamic models, where the dynamic behavior, due to parasitic capacitances and inductances of the device, is captured. The switching and conduction losses are subsequently estimated from the dynamic response of the system.

The same development approach was used as with the static models when integrating these variants together to ensure simple and fast swapping between model variants.

Table 1 summarizes the static and loss model variants as well as the number of possible model representations given those variants

The models described are used as the building blocks of more complex power electronics topologies. An example of the utilization of the models is presented in Figure 9.

The topology under investigation is an isolated DC-DC converter that represents the on-board charger of an EV. Using the models developed we were able to simulate the response of the converter when coupled with a simple PI controller that regulates the output current. Furthermore, we were also able to carry out an investigation on the impact of different semiconductor technologies on the performance of the charger. The output current and voltage curves, as well as the full-bridge semiconductor temperatures are provided in Figure 10, Figure 11, Figure 12 and Figure 13. 
Table 1. PowerElectronics library model variants

\begin{tabular}{lccc}
\hline & $\begin{array}{c}\text { Static } \\
\text { Variants }\end{array}$ & $\begin{array}{c}\text { Loss } \\
\text { Variants }\end{array}$ & $\begin{array}{c}\text { Total } \\
\text { Variants }\end{array}$ \\
\hline Diodes & 4 & 2 & 8 \\
IGBTs & 5 & 9 & 45 \\
MOSFETs & 5 & 9 & 45 \\
\hline
\end{tabular}

Moreover, we were able to use the developed system to execute batch simulations to estimate the impact of different switching frequencies on the Full-Bridge losses as presented in Figure 14. This capability to automatically execute batch simulations can be used in conjunction with Design of Experiments techniques to carry out system level parameter optimizations.

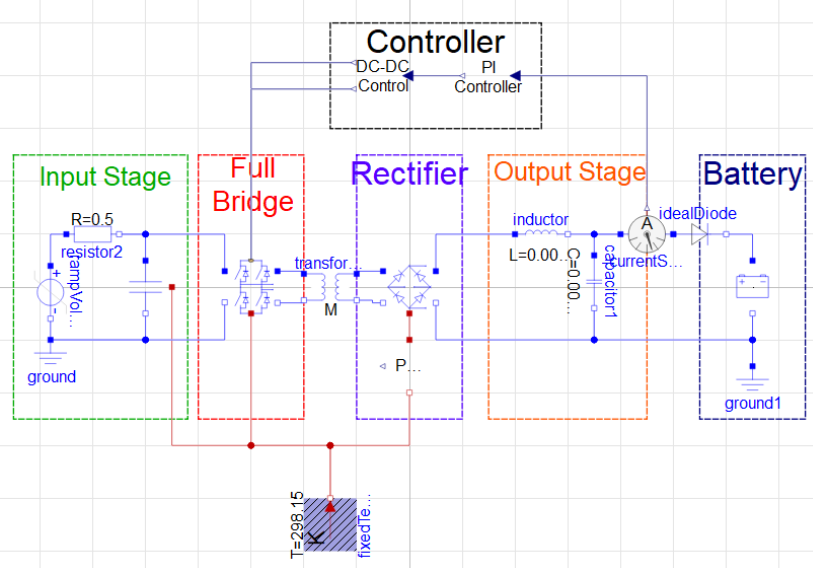

Figure 9. Hard-switched isolated DC-DC converter

Output Voltage
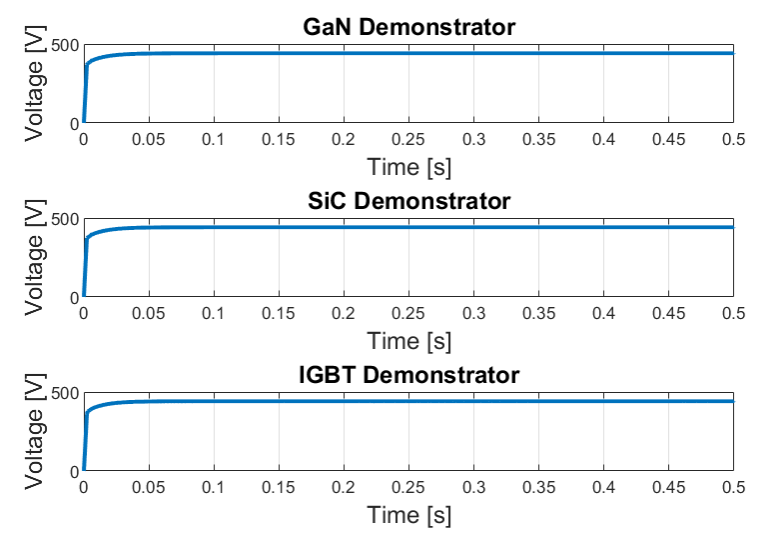

Figure 10. DC-DC Output voltage
Output Current
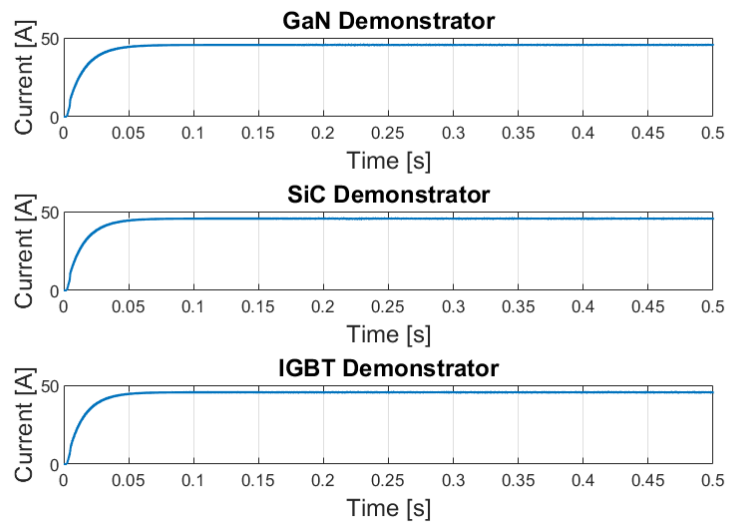

Figure 11. DC-DC Output current

Output Current Ripple

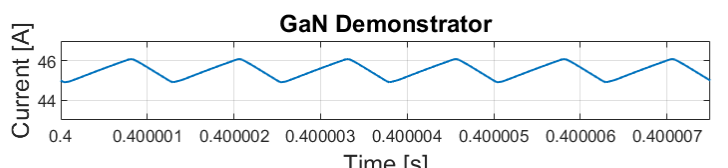

SiC Demonstrator
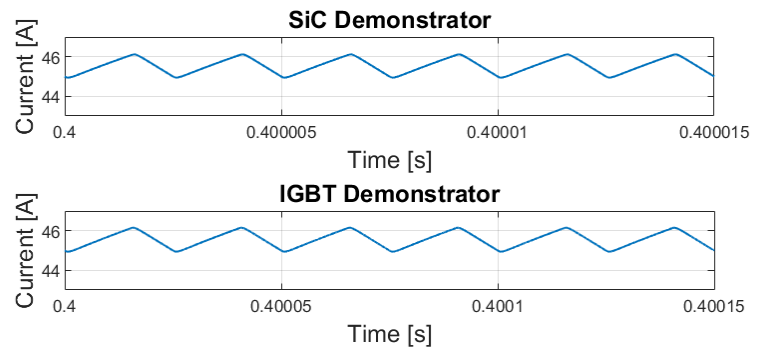

Figure 12. DC-DC Output current ripple

Full Bridge Semiconductor Temperature

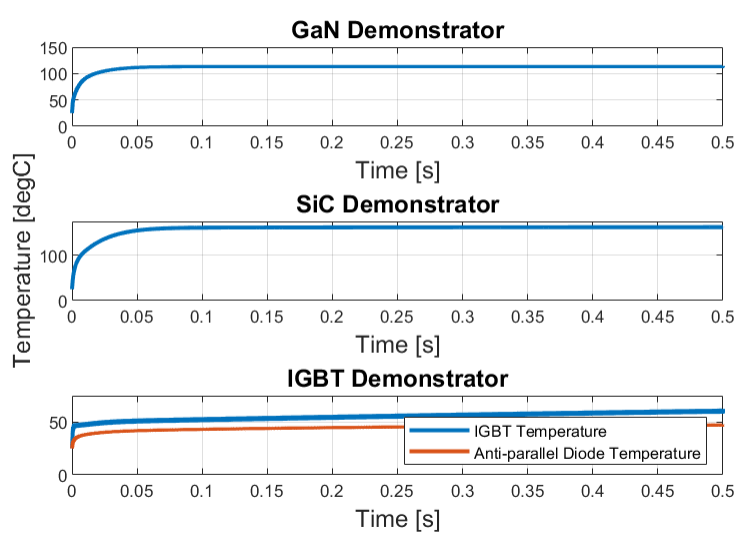

Figure 13. Full bridge semiconductor temperature 


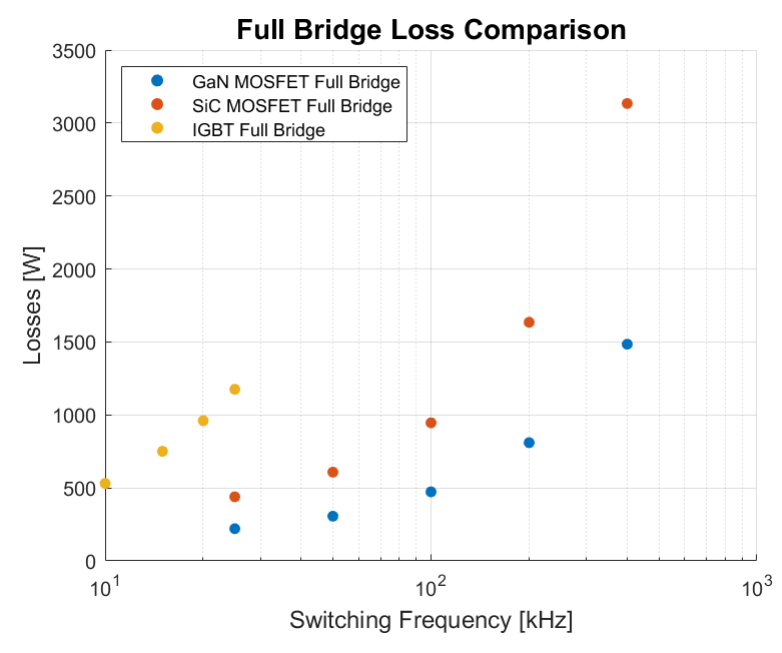

Figure 14. Full bridge technologies loss comparison

\subsection{EDU}

The electrical machines library found in the MSL (version 3.2.1) contains models for synchronous induction machines, including permanent magnet and synchronous reluctance motors. To develop the electrified powertrain digital twin, the Modelica models were used as templates and extended to form the EDU Modelica Library.

\subsubsection{Python Automated Data Importing}

The library is coupled with the Ricardo eMotor design database (eMAD, see Figure 15) using a Python script. The script automates data transfer and formatting and the process can be broken down as follows: 1) The Python script queries the motor requirement such as motor maximum power, DC voltage, etc. from the eMAD database 2) The user selects a specific MotorCAD design which meets the requirement. 3) The script extracts from eMAD detailed parameters such as d-q inductances, pole numbers, the open circuit voltage, nominal frequency etc. (see Table 2) and saves the result into a Modelica parameter file. 4) The user directly imports the file into Modelica using a function called Modelica.Utilities.Example.readReal Paramet er from MSL.

\subsubsection{EDU library structure}

Following the literature review, the SMPM and SMR motor types were considered as the initial focus for the EDU library. Referring to Figure 16, within the EmachineSummary package, a standard interface called MotorInterface was implemented as a partial model to define drive unit subsystem of the overall epropulsion system. In doing so, a user may choose between the various motor types, e.g., SMPM. Referring to Figure 17, three electrical pins (pin_p, pin_p1, pin_p2) are used to connect to a three-phase inverter. Motor voltage, current, torque and rotational speed signals connect through a Control Bus to the EDU controller via an external control bus. The torque output connects with a vehicle model (gear and drive model) using a rotational flange connector from the MSL. The thermal port releases heat due to the power losses from the motor, as explained in Section 2.3.3.

In the SMPMMotorTest and SMRMotorTest packages, the standard SMPM and SMR Modelica models were extended with the sub-component models based on outputs from MotorCAD. The modifications are mainly to improve the power loss equations (see the next section) to enhance correlation with MotorCAD data and to model the thermal behavior (see Section 2.3.3).

\subsubsection{Power loss models}

Firstly, using Ricardo's MotorCAD database material (e.g., see Figure 18), the generic power loss equations in Modelica were re-parameterized for a specific MotorCAD design, including stator core losses, stator winding losses, rotor winding losses and permanent magnet losses. The parameterization of the equations was performed using the MATLAB curve fitting toolbox and optimized to minimize the error across the range of operating motor speed and torque values. To achieve a better correlation with MotorCAD's data, some equations such as those for stator core losses were extended, for example, to include dependency on shaft torque. As MSL models do not specify an equation for the rotor core losses (lossPowerRotorCore is set to zero by default), a custom binomial equation as a function of motor speed and torque was included.

Through the ThermalAmbientSMPM interface block provided by Modelica, the motor power losses are extracted, and the detailed node temperatures are fed into the state-space thermal model. Temperatures corresponding to MotorCAD nodes ID 12 and 31 (the location nearest to the stator winding and permanent magnet, respectively) are used as temperature feedback signals to the thermal interface block.

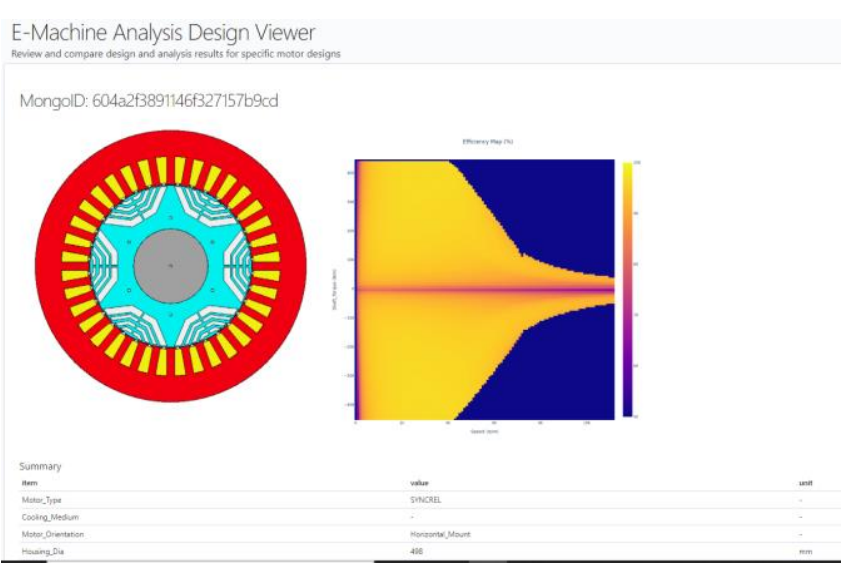

Figure 15. eMAD typical motor design 


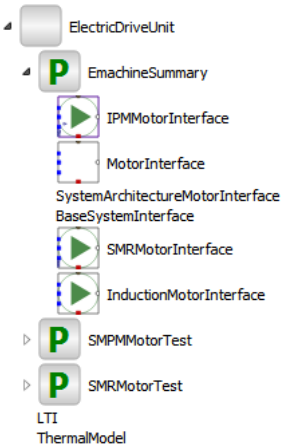

Figure 16. Structure of EDU library

Table 2. Modelica imported parameters from MotorCAD

\begin{tabular}{lcc}
\hline Parameters & Value & Unit \\
\hline Number of poles in pairs & 10 & \\
$\begin{array}{l}\text { Nominal frequency } \\
\text { Open circult voltage }\end{array}$ & 600 & $\mathrm{~Hz}$ \\
$\begin{array}{l}\text { Nominal stator resistance } \\
\text { per phase }\end{array}$ & 0.013 & $\Omega$ \\
$\begin{array}{l}\text { Stator main field } \\
\text { inductance in d-axis }\end{array}$ & 0.1755 & $\mathrm{mH}$ \\
$\begin{array}{l}\text { Stator main field } \\
\text { inductance in q-axis }\end{array}$ & 0.6618 & $\mathrm{mH}$ \\
$\begin{array}{l}\text { Stator copper loss } \\
\text { Stator iron loss } \\
\text { Magnet loss }\end{array}$ & $\begin{array}{l}\text { MotorCAD design map } \\
\text { Rotor iron loss }\end{array}$ & $\begin{array}{l}\text { MotorCAD design map } \\
\text { MotorCAD design map } \\
\text { MotorCAD design map }\end{array}$ \\
\hline
\end{tabular}

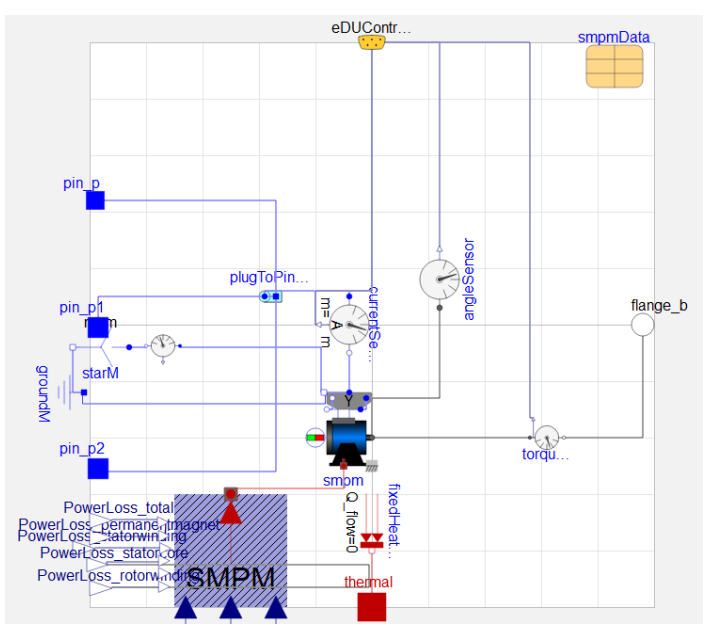

Figure 17. IPM_motor_interface

\subsubsection{Thermal model}

In order to better represent the thermal behavior of the EDU, a reduced order state-space thermal model (see Figure 19 and Figure 20) was also developed for Modelica and parameterized with MotorCAD data files, namely “.cmf" files for thermal capacitances, ".rmf" files for thermal resistances, ".pmf” files for power losses, ".tmf" files for node temperatures.

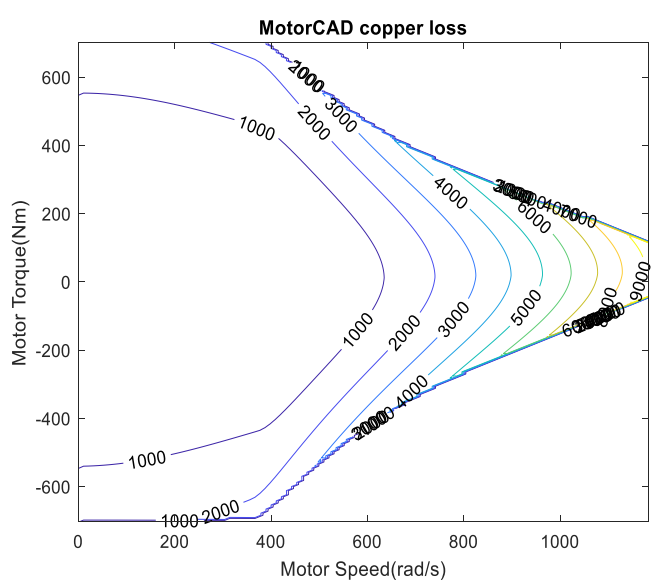

Figure 18. Example stator copper loss map from MotorCAD
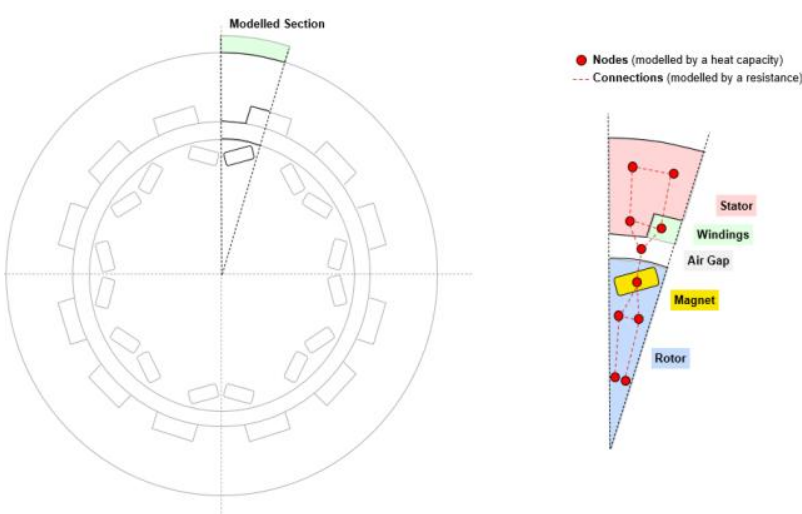

Figure 19. A guide to reduced lumped-mass thermal models in MotorCAD

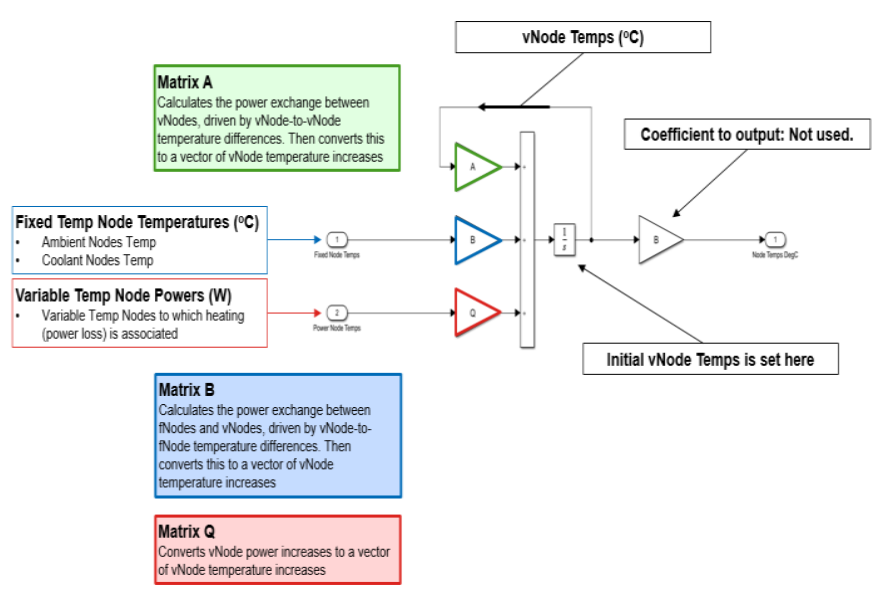

Figure 20. Motor node temperature calculation 


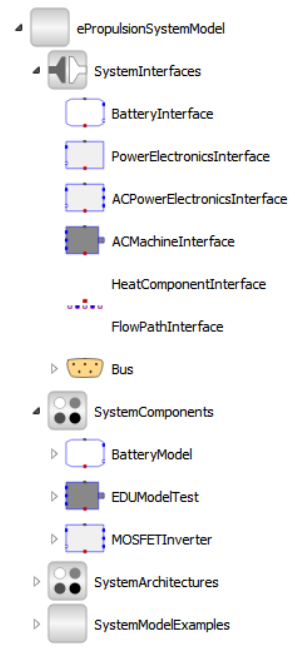

Figure 21. The structure of the ePropulsionSystemModel

\section{4 ePropulsionsystemModel}

The ePropulsionsystemmodel library was built by using the components in Battery, PowerElectronics and EDU sub-libraries, and its structure is shown in Figure 21. The interfaces for the key components (Battery, PowerElectronics and EDU) in the ePropulsionSystemModel library were created in the SystemInterfaces package. Then the replaceable models of each of these components were built in the SystemComponents package by using the interfaces and the models developed in Battery, PowerElectronics and EDU sub-libraries. By connecting the replaceable Battery model, EDU model and Inverter model, an ePropulsion System Architecture system model can be created. Figure 22 shows an example model from the SystemArchitectures package which is an ePropulsion system model with Alternating Current (AC) architecture. Because the Inverter and EDU are replaceable, by choosing different Inverter and EDU models, different types of ePropulsion systems can be created. In this way, the ePropulsion system model can work as a standalone model or be integrated into complete vehicle models.

\section{Use Case}

To assess the ePropulsion system library, a vehicle cosimulation was done. The ePropulsion system library was imported into Ricardo IGNITE, which is a physics-based tool developed for complete vehicle system modelling and simulation. IGNITE features comprehensive built-in automotive Modelica libraries. These enable users not only to quickly and accurately model conventional and highly complex vehicle system models including hybridelectric, full electric and novel vehicles, but also to import any Modelica based library such as the ePropulsion system library.

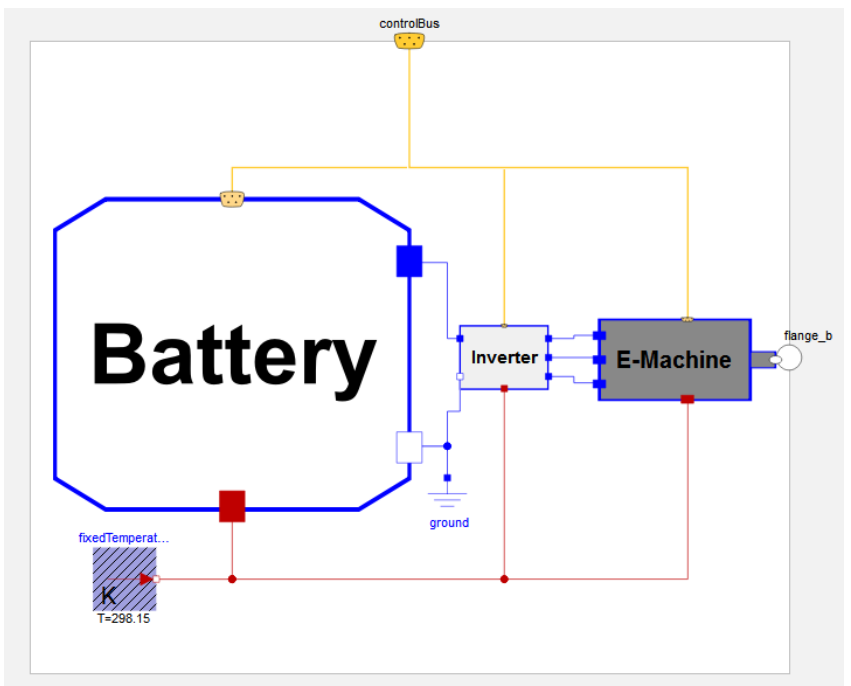

Figure 22. The ePropulsion system model with AC Architecture

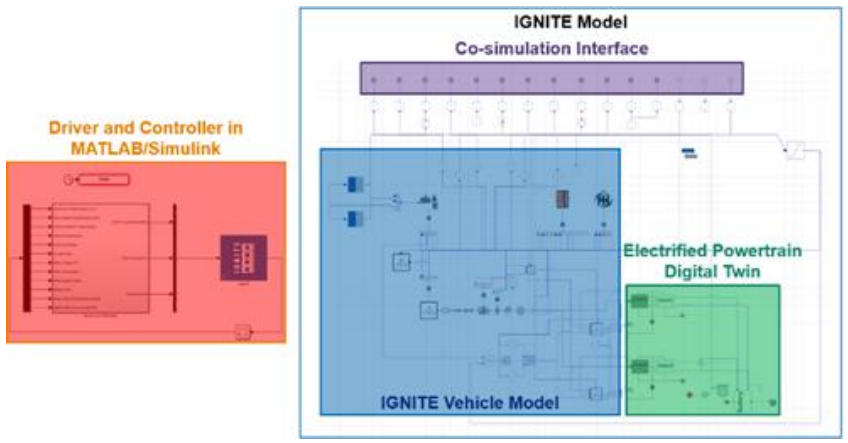

Figure 23. The co-simulation models

Figure 23 shows the co-simulation models used in this study. There are three important parts of the IGNITE model. These are the IGNITE vehicle model, the electrified powertrain digital twin and the co-simulation interface. The IGNITE vehicle model was built with the built-in, comprehensive vehicle modelling libraries. The electrified powertrain digital twin was built by using the components from the ePropulsion system library. The cosimulation interface provided the input and output interfaces for co-simulation. The vehicle controller used was developed in MATLAB/Simulink and coupled to the Modelica libraries, as shown in Figure 23, to showcase the ability to utilize pre-existing controllers developed in other environments. As this co-simulation model is a forward-facing simulation, a driver model was developed and included in MATLAB/Simulink as well.

The vehicle used in this study is a $6 \times 4$ long haul truck model which was defined based on typical MY2019 specifications. The key vehicle parameters are shown in Table 3. The vehicle performance attributes and propulsion system requirements are also given in Table 4 . 
Table 3. Vehicle parameters

\begin{tabular}{lcc}
\hline Parameter & Value & Unit \\
\hline GVW & 44 & $\mathrm{t}$ \\
Cd & 0.6 & - \\
Frontal area & 10.2 & $\mathrm{~m} 2$ \\
Tyre RRC & 6.4 & $\mathrm{~N} / \mathrm{kN}$ \\
Tyre rolling radius & 0.49 & $\mathrm{~m}$ \\
\hline
\end{tabular}

Table 4. Vehicle performance attributes and propulsion system requirements

\begin{tabular}{cc}
\hline $\begin{array}{c}\text { Vehicle Performance } \\
\text { Attributes }\end{array}$ & $\begin{array}{c}\text { Propulsion System } \\
\text { Requirements }\end{array}$ \\
\hline $90 \mathrm{~km} / \mathrm{h}$ on $3 \%$ grade & Powertrain max \\
$40 \mathrm{~km} / \mathrm{h}$ on $10 \%$ grade & continuous power \\
Climbing $30 \%$ grade & $\geq 525 \mathrm{~kW}$ \\
$6 \times 2$ mode for GCW $<32 \mathrm{t}$ & $6 \times 2$ mode max \\
(2nd axle lifted) & continuous power \\
& $\geq 380 \mathrm{~kW}$ \\
Min top speed $120 \mathrm{~km} / \mathrm{h}$ & Powertrain max \\
on flat road & $\geq 67,500 \mathrm{Nm}$ ('at \\
& wheels') \\
\hline
\end{tabular}

A baseline configuration of the propulsion system was defined which has an $800 \mathrm{~V}$ system voltage, two EDUs, and one battery pack which has $530 \mathrm{kWh}$ of nominal useable energy. To evaluate the baseline propulsion system performance, simulations were carried out using a variety of drive cycles.

\section{Simulation Results}

The drive cycle target speed profile is presented in Figure 24 alongside the actual vehicle speed. Using the imported controller and the Digital Twin models we are able to accurately match the actual speed with the target profile.

Having achieved the desired speed profile, we can then extract curves to assess the performance of each part of the electrified powertrain. Specifically, in Figure 25 the battery voltage, load current, delivered power and SoC are presented for the drive cycle. From those curves we can extract metrics such as depth of discharge and charge throughput for a single cycle, as well as total regeneration energy for that cycle.

In Figure 26 the inverter power losses and output phase currents are plotted, enabling us to evaluate if the semiconductor peak currents are within the absolute rating limits, as well as compare the inverter losses with the total delivered power by the battery.

Finally $_{2}$ Figure 27 and Figure 28 present curves extracted from a single eMachine model. In Figure 27 the eMachine torque and speed are plotted, which closely follow the controller demanded speed and torque, while in Figure 28 the different loss components, as well as the total losses of the eMachine are presented, enabling us to evaluate the performance of the machine.

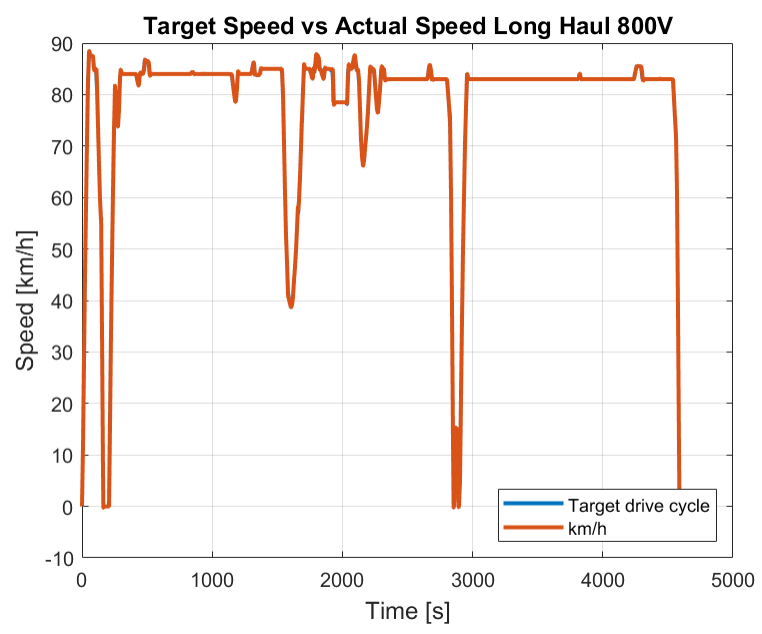

Figure 24. Target vs Actual Speed

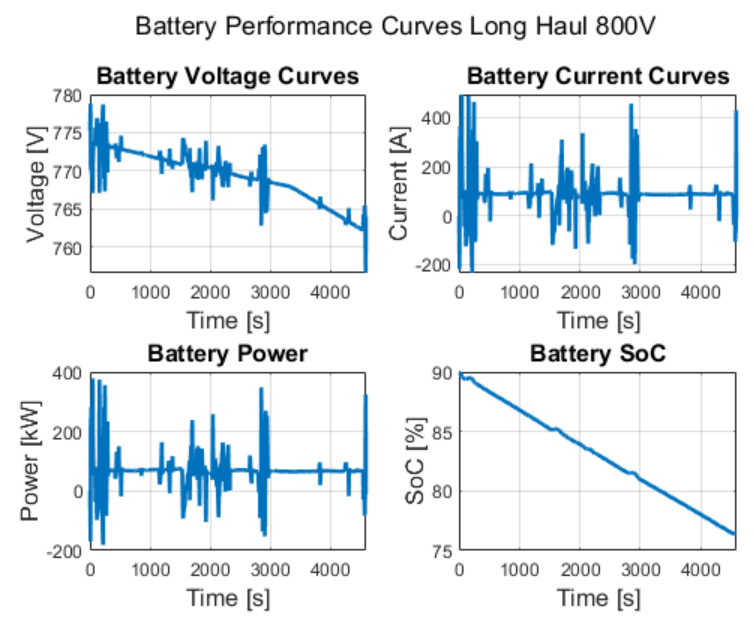

Figure 25. Battery Performance Curves
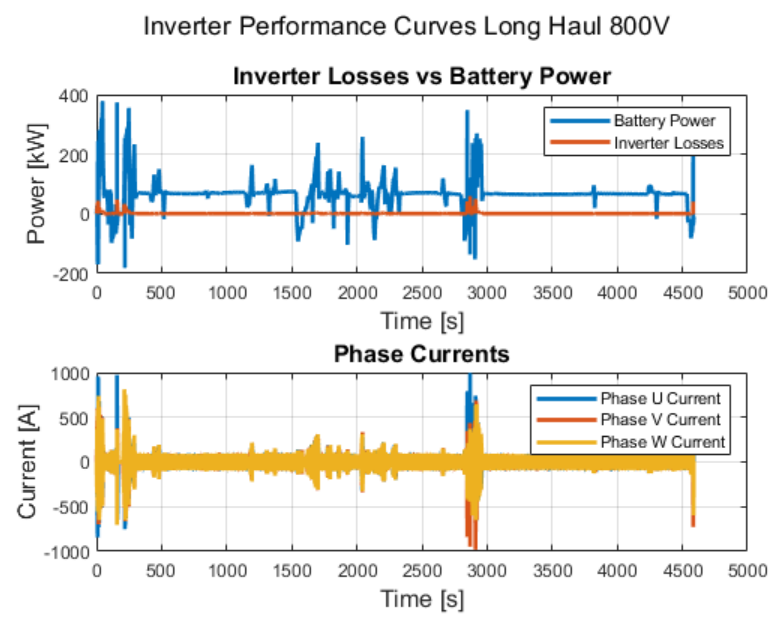

Figure 26. Inverter Performance Curves 


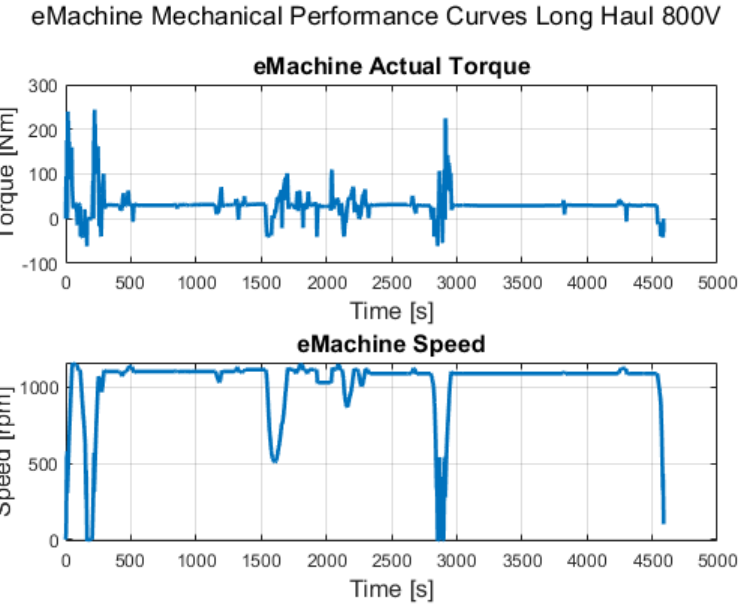

Figure 27. eMachine Mechanical Performance Curves

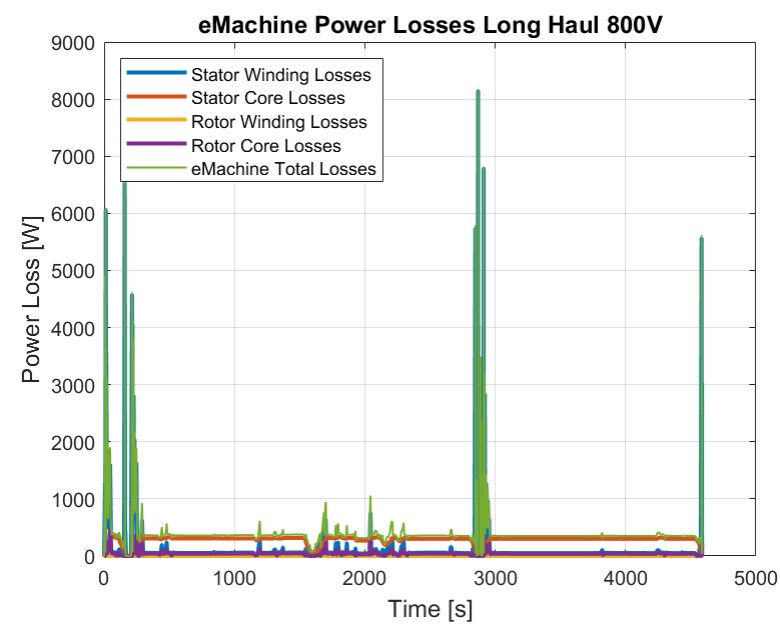

Figure 28. eMachine Power Losses

\section{Total Cost of Ownership (TCO) Assessment}

Optimising journey time and cost is the most important criteria for road-freight vehicles. To assess the impact of powertrain design and configuration on this criterion, a TCO study was carried out. The TCO study assessed the overall cost of vehicle ownership as $€ /$ t.100km and collected a wide range of inputs, both from the detailed system simulation previously discussed, expected degradation, component costs and costs of operation (electricity prices etc). The TCO model is presented schematically in Figure 29.

The following assumptions have been made for the purpose of the TCO assessment.

Table 5. TCO model assumptions

\begin{tabular}{lcc}
\hline Parameter & Value & Unit \\
\hline Electricity Price & 0.25 & $€ / \mathrm{kWh}$ \\
Battery Pack Cost & 200 & $€ / \mathrm{kWh}$ \\
Power Electronics and & 43 & $€ / \mathrm{kWh}$ \\
E-motor Cost & & \\
\hline
\end{tabular}

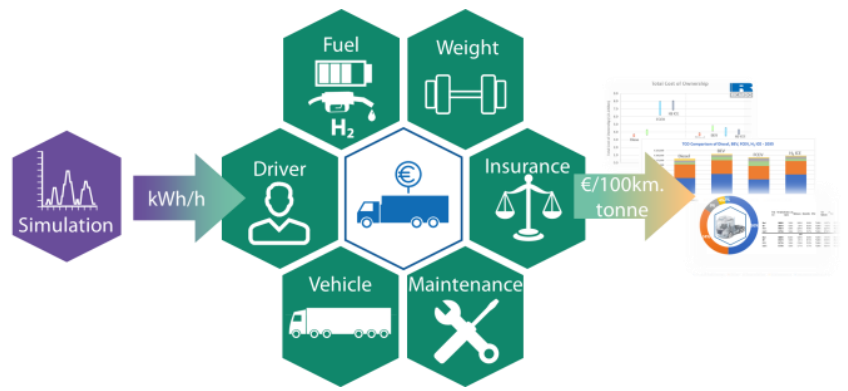

Figure 29. Schematic overview of Total Cost of Ownership model

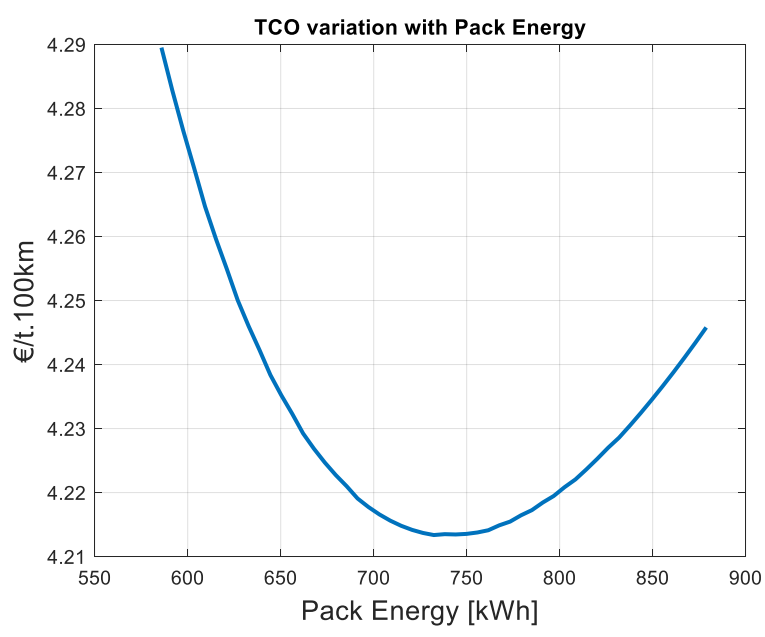

Figure 30. TCO sensitivity to battery pack energy

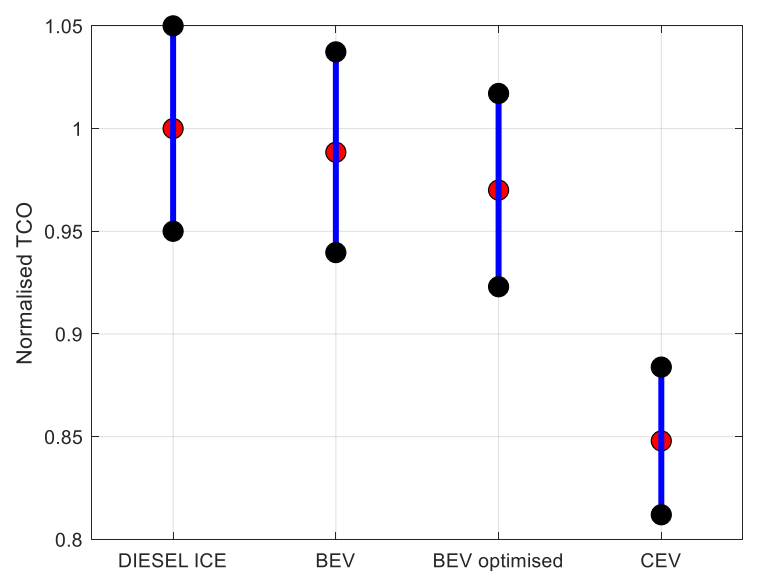

Figure 31. Comparison of TCO for different powertrain technologies

The TCO model enables a what-if analysis on the impact of various powertrain parameters (or indeed external parameters) on TCO. An example is shown in Figure 30 where the TCO sensitivity to battery pack installed energy is presented. A clear minimum can be seen which suggests that, for this vehicle application, it is beneficial to have a larger battery pack installed, which improves battery lifetime and range. 
The TCO model is also used to compare different powertrain technologies as shown in Figure 31. In this example, the TCO for a Diesel Internal Combustion Engine (ICE) is used as a baseline and compared against a BEV, optimised BEV (using the initial results from above) and a Catenary Electric Vehicle (CEV) variant. A detailed comparison, including Fuel Cell EV will be the focus of a future study.

\section{Conclusion}

The Modelica library of electrified powertrain components, ePropulsionsystem, the sub-libraries of key sub-components (Battery, Power Electronics and EDU), and the various models included in the sublibraries are detailed in the paper. The ePropulsionsystem library enables rapid construction of Digital Twins for Battery, EDU and Power Electronics systems in an electrified powertrain, and provides an approach which can scale automatically from low-fidelity for fast system level simulation to high-fidelity for subsystem design analysis in the electrified powertrain development process. An example of applying the ePropulsionSystem library to an EV use case is also discussed. In the use case, the co-simulation models and driving cycles simulation result are presented. Then, by using the co-simulation models, a Total Cost of Ownership (TCO) optimisation is discussed as an example to prove that the developed Modelica library can be used to assess and optimise a wide range of electrified propulsion architectures.

\section{Acknowledgements}

This work has been supported by Innovate UK (Project No: 81341). The authors would also like to thank Milan Cvetkovic and Dmitry Rozhdestvenskiy for their support with implementation of the software.

\section{References}

AG, I. T. (2020). Transient thermal measurements and thermal. Munich, Germany: Infineon Technologies AG.

Andrea Cordoba-Arenas, S. O. (2015). "A control-oriented lithium-ion battery pack model for plug-in hybrid electric vehicle cycle-life studies and system design with consideration of health management". In: Journal of Power Sources, Vol. 279, pp. 791-808.

Bao, R., Fotias, N., \& McGahan, P. (2021). "Using Virtual Product Development with Design of Experiments to Design Battery Packs for Electrified Powertrain". In: SAE Technical Paper 2021-01-0764.

Bazzi, A. M. (2013). "Electric machines and energy storage technologies in EVs and HEVs for over a century". In: International Electric Machines \& Drives Conference. Chicago, IL, USA: IEEE.

Cellier, F. E., Clauß, C., \& Urquía, A. (2007). "Electronic circuit modeling and simulation in Modelica". In: Proc. 6th EUROSIM Congress on Modelling and Simulation. Ljubljana, Slovenia.
Ceraolo, M. (2015). "A new Modelica Electric and Hybrid Power Trains library". In: Proceedings of the 11th International Modelica Conference, Versailles, September 21-23 (pp. 785-794). France: Linköping Electronic Conference Proceedings.

Chen, M., \& Rincon-Mora, G. A. (2006). "Accurate electrical battery model capable of predicting runtime and I-V performance". In: IEEE Transactions on Energy Conversion, Vol: 21(2), pp. 504-511.

Dao, T.-S., \& Schmitke, C. (2015). "Developing Mathematical Models of Batteries in Modelica for Energy Storage Applications". In: The 11th International Modelica Conference.

Denz, P., Schmitt, T., \& Andres, M. (2014). "Behavioral Modeling of Power Semiconductors in Modelica". In: Proceedings of the 10th International ModelicaConference, (S. 343-352). Lund, Sweden.

Dorrell, D. G., Knight, A. M., Popescu, M., Evans, L., \& Staton, D. A. (2010). "Comparison of different motor design drives for hybrid electric vehicle". In: IEEE Energy Conversion Congress and Exposition. Atlanta, GA, USA: IEEE.

Einhorn, M., Conte, F. V., Kral, C., Niklas, C., Popp, H., \& Fleig, J. (2011). "A Modelica Library for Simulation of Elecric Energy Storages". In: The 8th International Modelica Conference.

Einhorn, M., F.V.Conte, C.Kral, C.Niklas, H.Popp, \& J.Fleig. (2111). "A Modelica Library for Simulation of Electric Energy Storages". In: Proceedings of the 8th International Modelica Conference; March 20th-22nd; (pp. 436-445). Dresden: Linköping University Electronic Press; Linköpings universitet.

Fan, G., Pan, K., Bartlett, A., Canova, M., \& Rizzoni, G. (2014). "Electrochemical-Thermal Modeling of Li-Ion Battery Packs". In: ASME 2014 Dynamic Systems and Control Conference. San Antonio, Texas, USA.

Gerl, J., Janczyk, L., Krüger, I., \& Modrow, N. (2014). "A Modelica Based Lithium Ion Battery Model". In: The 10th International ModelicaConference.

Gragger, J. V., Kral, H. G., Hansjörg, T. B., \& Pirker, K. F. (2006). "The SmartElectricDrives Library - Powerful Models for Fast".In: Modelica Conference 2006 at arsenal research in Austria. Vienna: The Modelica Association .

Guo, M., Jin, X., \& White, R. E. (2017). "An Adaptive ReducedOrder-Modeling Approach for Simulating Real-Time Performances of Li-Ion Battery Systems". In: Journal of The Electrochemical Society, Vol: 164(14), pp. A3602-A3613.

Haumer, A., \& Kral, C. (2012). "Motor Management of Permagnent Magnet Synchronous Machines". In: Proceedings of the 9th International Modelica Conference. Munich: Modelica.

Haumer, A., Kral, C., Kapeller, H., Bäuml, T., \& Gragger, J. V. (2009). "The Advanced Machines Library". In: Proceedings 7th Modelica Conference, Italy, Sep. 20-22. Como: Modelica.

He, H., Xiong, R., \& Fan, J. (2011). "Evaluation of Lithium-Ion Battery Equivalent Circuit Models for State of Charge Estimation by an Experimental Approach". In Energies, Vol: 4(4), pp. 582-598.

Hwang, M.-H., Han, J.-H., Kim, D.-H., \& Cha, H.-R. (2018). "Design and Analysis of Rotor Shapes for IPM Motors in EV Power Traction Platforms". Energies, vol: 2601.

Johnson, V. H., Pesaran, A. A., \& Sack, T. (2000). "Temperature- Dependent Battery Models for High-Power 
Lithium-Ion Batteries". In: 17th Annual Electric Vehicle Symposium. Montreal, Canada.

Kral, C., \& Haumer, A. (2011). "The New FundamentalWave Library for Modeling Rotating Electrical Three Phase Machines". In: Proceedings of the 8th International Modelica Conference; March 20th-22nd; Technical Univeristy (pp. 170-179). Dresden: Linköping University Electronic Press; Linköpings universitet.

Lai, S. C., Hill, C. I., \& Suchato, N. (2019). "Implementation of an Advanced Modelica Library for Evaluation of Inverter Loss Modeling". In: 2019 IEEE Texas Power and Energy Conference (TPEC). College Station, TX, USA.

Lee, B. H., Kim, K.-S., Jung, J.-W., Hong, J. P., \& Kim, Y. K. (2012). "Temerature Estimation of IPMSM using Thermal Equivalent Circult". In: IEEE Transactions of magnetics, Vol. 48, No.11.

McDonald, D. (2012). "Electric Vehicle Drive Simulation with MATLAB/Simulink". In: proceedings of the 2012 NorthCentral Section Conference. American Society for Engineering Education .

McGahan, P., Rouaud, C., \& Booker, M. (2019). "Comparison of Model Order Reduction Techniques for Real-Time Battery Thermal Modelling". In: SAE Technical Paper 2019-010503. doi:10.4271/2019-01-0503

Mohd, T. A., Hassan, M. K., \& Aziz, W. M. (2015). "Mathematical modeling and simulation of an electric vehicle". In: Journal of Mechanical Engineering and Sciences (JMES), pp. 1312-1321.

Nelson, P., Dees, D., Amine, K., \& Henriksen, G. (2002). "Modeling thermal management of lithium-ion PNGV batteries". In: Journal of Power Sources, Vol: 110(2), pp.349356.

Park, C., \& Jaura, A. K. (2003). "Dynamic Thermal Model of LiIon Battery for Predictive Behavior in Hybrid and Fuel Cell Vehicles". In: SAE Technical Paper 2003-01-2286. doi:10.4271/2003-01-2286

Perez, H., Shahmohammadhamedani, N., \& Moura, S. (2015). "Enhanced Performance of Li-Ion Batteries via Modified Reference Governors and Electrochemical Models". In: IEEE/ASME Transactions on Mechatronics, 20(4), 15111520.

Pesaran, A. A. (2002). "Battery thermal models for hybrid vehicle simulations". In: Journal of Power Sources, Vol: 110(2), pp. 377-382.

Qin, D., Li, J., Wang, T., \& Zhang, D. (2019). "Modeling and Simulating a Battery for an Electric Vehicle Based on Modelica". In: Automotive Innovation, Vol: 2(3), pp. 169177.

Ramakrishnan, K., Stipetic, S., Gobbi, M., \& Mastinu, G. (2018). "Optimal Sizing of Traction Motors Using Scalable Electric Machine Model". In: IEEE Transactions on Transportation Electrification, Vol: 4 , no. 1.

Surewaard, E., Karden, E., \& Tiller, M. (2003). "Advanced Electric Storage System Modeling in Modelica". In: The 3rd International Modelica Conference.

Urkizu, J., Mazuela, M., Alacano, A., Aizpuru, I., Chakraborty, S., Hegazy, O., Klink, R. (2019). "Electric Vehicle Inverter Electro-Thermal Models Oriented to Simulation Speed and Accuracy Multi-Objective Targets". In: Energies.

Vetter, J., Novák, P., Wagner, M. R., Veit, C., Möller, K.-C., Besenhard, J. O., Hammouche, A. (2005). "Ageing mechanisms in lithium-ion batteries". In: Journal of Power Sources, Vol: 147(1-2), pp. 269-281.

Xing, Sun, J., \& Lei. (2014). "Parameterization of Three-Phase Electric Machines Models for EMI sIMULATION". In: IEEE Transactions on power electronics, Vol: 29 , No. 1. 